\title{
SEMICONCAVE CONTROL-LYAPUNOV FUNCTIONS AND STABILIZING FEEDBACKS*
}

\author{
LUDOVIC RIFFORD ${ }^{\dagger}$
}

\begin{abstract}
We study the general problem of stabilization of globally asymptotically controllable systems. We construct discontinuous feedback laws, and particularly we make it possible to choose these continuous outside a small set (closed with measure zero) of discontinuity in the case of control systems which are affine in the control; moreover this set of singularities is shown to be repulsive for the Carathéodory solutions of the closed-loop system under an additional assumption.
\end{abstract}

Key words. asymptotic controllability, control-Lyapunov function, feedback stabilization, nonsmooth analysis

AMS subject classifications. 93D05, 93D20, 93B05, 34D20, 49J52, 49L25, 70K15

PII. S0363012900375342

1. Introduction. In a previous paper [23] we considered the stabilization problem for standard control systems. In particular, we proved that if a control system is globally asymptotically controllable, then one can associate to it a control-Lyapunov function which is semiconcave outside the origin. The goal of this article is to show the utility of the semiconcavity of such functions in the construction of stabilizing feedbacks.

We consider a standard control system of the general form $\dot{x}=f(x, u)$ which is globally asymptotically controllable, our objective being to design a feedback law $u: \mathbb{R}^{n} \rightarrow U$ such that the origin of the closed-loop system $\dot{x}=f(x, u(x))$ is globally asymptotically stable. Unfortunately, as pointed out by Sontag and Sussmann [28] and by Brockett [7], a continuous stabilizing feedback fails to exist in general. In addition to that, a smooth Lyapunov function may not exist either. As a matter of fact, although smooth Lyapunov-like techniques have been successfully used in many problems in control theory, it was shown by many authors (see Artstein [5] for the affine case, and more recently Clarke, Ledyaev, and Stern [11] for the general case) that there is no hope of obtaining a smooth Lyapunov function in the general case of globally asymptotically controllable systems. (The existence of such a function is indeed equivalent to that of a robust stabilizing feedback; see [17, 21].) These facts lead us to consider nonsmooth control-Lyapunov functions and particularly semiconcave control-Lyapunov functions; we proved the existence of such a function in our previous article [23]. This article builds on this result to derive a useful and direct construction of stabilizing feedbacks. In fact, the semiconcavity of the control-Lyapunov function allows us to give an explicit formula for the design of the stabilizing feedbacks. More particularly, this formula can be used in the context of control systems which are affine in the control to extend Sontag's formula [26] to the case of discontinuous feedback laws. Furthermore, the main result of this paper asserts that when the control system is affine in the control, we can design a feedback which is continuous on an open dense set and which stabilizes the closed-loop system in the sense of Carathéodory solutions. Surprisingly, we show that in this case, under an additional assumption on

\footnotetext{
*Received by the editors July 14, 2000; accepted for publication (in revised form) February 1, 2002; published electronically July 24, 2002.

http://www.siam.org/journals/sicon/41-3/37534.html

${ }^{\dagger}$ Institut Girard Desargues, Université Lyon I, 43 Bd du 11 Novembre 1918, 69622 Villeurbanne Cedex, France (rifford@igd.univ-lyon1.fr).
} 
the control-Lyapunov function, all the trajectories of the closed-loop system remain in the set of continuity for positive times; in other words, the set of singularities of the stabilized system is repulsive, and hence the feedback law is continuous (even locally Lipschitz) along the trajectories for $t>0$.

Our paper is organized as follows: In section 2 we describe our main results. In section 3 we present some basic facts about nonsmooth analysis, semiconcavity, and discontinuous stabilizing feedbacks. In sections 4 and 5 , we give the proofs of different results. Finally, the main theorems are proved in sections 6 and 7 .

Throughout this paper, $\mathbb{R}_{\geq 0}$ denotes the nonnegative reals, $\|\cdot\|$ a norm on $\mathbb{R}^{n}$, $B$ the open ball $B(0,1):=\{x:\|x\|<1\}$ in $\mathbb{R}^{n}$, and $\bar{B}$ the closure of $B$.

\section{Definitions and statements of the results.}

2.1. General control systems. We study systems of the general form

$$
\dot{x}(t)=f(x(t), u(t)),
$$

where the state $x(t)$ takes values in a Euclidean space $\mathbb{R}^{n}$, the control $u(t)$ takes values in a given compact metric space $U$, and $f$ is locally Lipschitz in $x$ uniformly in $u$. We distinguish a special element " 0 " in $U$ and assume that the state $x=0$ is an equilibrium point (i.e., $f(0,0)=0$ ). We are interested in globally asymptotically controllable systems, which we proceed to define.

DEFINITION 2.1. The system (2.1) is globally asymptotically controllable (GAC) if there exists a nondecreasing function

$$
\tilde{\theta}: \mathbb{R}_{\geq 0} \rightarrow \mathbb{R}_{\geq 0}
$$

such that $\lim _{r \rightarrow 0^{+}} \tilde{\theta}(r)=0$, with the property that, for each $\xi \in \mathbb{R}^{n}$, there exist a control $u: R_{\geq 0} \rightarrow U$ and a corresponding trajectory $x(\cdot): \mathbb{R}_{\geq 0} \rightarrow \mathbb{R}^{n}$ such that $x(0)=\xi$,

$$
x(t) \rightarrow 0 \text { as } t \rightarrow \infty,
$$

and

$$
\sup \{\|x(t)\|: 0 \leq t<\infty\} \leq \tilde{\theta}(|\xi|) .
$$

This definition of global asymptotic controllability is appropriate under the assumption of compactness of the control set $U$. When this set is not compact, we must add some conditions on the open-loop controls which stabilize the initial states; we refer to the papers of Sontag and Sussmann [29, 30] for a generalization of this definition to the general case on a noncompact control set.

REMARK 2.2. This definition seems weaker than the one given initially in [23]. However, as explained by Sontag and Sussmann in [29, 30], a routine argument involving continuity of trajectories with respect to initial states shows that our different definitions are indeed equivalent.

Our objective is to design a feedback law $u: \mathbb{R}^{n} \rightarrow U$ such that the origin of the closed-loop system (2.1) is globally asymptotically stable; that is, such that the new system

$$
\dot{x}(t)=f(x(t), u(x(t)))
$$


is globally asymptotically stable. Our method relies on nonsmooth Lyapunov functions, which we proceed to define; we refer to the next section for the definition of the proximal subdifferential $\partial_{P} V(\cdot)$.

DEFINITION 2.3. A control-Lyapunov function for the system (2.1) is a continuous function $V: \mathbb{R}^{n} \rightarrow \mathbb{R}$ which is positive definite (i.e., $V(0)=0$ and $V(x)>0$ for $x \neq 0$ ), proper (i.e., $V(x) \rightarrow \infty$ when $\|x\| \rightarrow \infty$ ), and such that there exists a positive definite continuous function $W: \mathbb{R}^{n} \longrightarrow \mathbb{R}$ with the property that, for each $x \in \mathbb{R}^{n} \backslash\{0\}$, we have

$$
\forall \zeta \in \partial_{P} V(x), \quad \min _{u \in U}\langle\zeta, f(x, u)\rangle \leq-W(x) .
$$

The present article is based on the following theorem, which is a refinement of a result proved in [23]. The regularity of our control-Lyapunov function will be crucial for the construction of discontinuous stabilizing feedbacks.

THEOREM 2.4. If the system (2.1) is GAC, then there exists a control-Lyapunov function $V$ which is semiconcave on $\mathbb{R}^{n} \backslash\{0\}$ and such that

$$
\forall x \in \mathbb{R}^{n} \backslash\{0\}, \forall \zeta \in \partial_{P} V(x), \min _{u \in U}\langle\zeta, f(x, u)\rangle \leq-V(x) .
$$

This theorem differs from the one given in our previous article [23] in the decrease condition (2.4). Here, we assert that we can take $V$ as the function $W$ of Definition 2.3. This special form of the infinitesimal decrease condition (2.4) will allow us to obtain exponential decrease for $V(x(t))$ and will make it possible to give closed-form estimates (in terms of $V$ ) on the rate of stabilization.

Now, using the concept of $\pi$-trajectories and of Euler trajectories which will be defined in the next section, we give a general result on the existence of stabilizing feedbacks; this result was announced in [25].

TheOREM 2.5. Assume that the system (2.1) is GAC. Then there exists a feedback $u: \mathbb{R}^{n} \rightarrow U$ for which the system $\dot{x}=f(x, u(x))$ is globally asymptotically stabilizable in the sense of $\pi$-trajectories and in the Euler sense.

Moreover, if we consider a control-Lyapunov function $V$ for the given system, then the stabilizing feedback can be designed as follows:

- We set $u(0)=0$.

- For each $x \in \mathbb{R}^{n} \backslash\{0\}$, we choose arbitrarily $\zeta \in \partial_{L} V(x)$ and we set $u:=u(x) \in U$, where $u(x)$ is any point in $U$ such that $\langle\zeta, f(x, u)\rangle \leq-W(x)$.

Furthermore, if the control-Lyapunov function $V$ is the one given by Theorem 2.4 (i.e., if $W=V$ ), then we have

$$
V(x(t)) \leq e^{-t} V\left(x_{0}\right)
$$

for any Euler trajectory starting at $x_{0} \in \mathbb{R}^{n}$.

The existence of a discontinuous feedback which is stabilizing in the sense of the $\pi$-trajectories is not new; it appeared initially in the article of Clarke et al. [10]. We refer also to Ancona and Bressan [4], who proved a slightly stronger result in the sense that their feedback stabilizes the closed-loop system in the sense of Carathéodory; their proof does not use nonsmooth control-Lyapunov functions. However, here the consideration of a semiconcave control-Lyapunov function leads to a simple proof and allows us to give an explicit formula for the design of the feedback. Moreover, we are able to design some stabilizing feedbacks which are rather regular in the case of affine systems. 
2.2. Affine control systems. Let us assume now that the control system is affine in the control, that is,

$$
f(x, u)=f_{0}(x)+\sum_{i=1}^{m} u_{i} f_{i}(x) \quad \forall(x, u) \in \mathbb{R}^{n} \times U,
$$

where the $f_{0}, \ldots, f_{m}$ are locally Lipschitz functions from $\mathbb{R}^{n}$ into $\mathbb{R}^{n}$ and where $U$ is a strictly convex and compact set of $\mathbb{R}^{m}$.

REMARK 2.6. Instead of assuming the control set $U$ to be strictly convex, we could make a weaker assumption of convexity. As a matter of fact, if the control set is supposed to be convex, we can define a subset of it which is strictly convex and for which the control system (2.6) keeps the same properties of controllability. Consequently all our results hold in the case of a convex compact control set.

First of all, assuming the knowledge of a control-Lyapunov function, we are able to give an explicit feedback law; it reduces to Sontag's formula [26] in the smooth case.

THEOREM 2.7. Assume that $V$ is a control-Lyapunov function for (2.6) and consider any selection $\zeta_{V}(\cdot)$ of $\partial_{L} V(\cdot)$. Then the feedback control defined by

$$
u_{i}(x):=-\phi\left(\left\langle f_{0}(x), \zeta_{V}(x)\right\rangle, \sum_{i=1}^{m}\left\langle f_{i}(x), \zeta_{V}(x)\right\rangle^{2}\right)\left\langle f_{i}(x), \zeta_{V}(x)\right\rangle,
$$

where

$$
\phi(a, b)=\left\{\begin{array}{cc}
\frac{a+\sqrt{a^{2}+b^{2}}}{b} & \text { if } b \neq 0, \\
0 & \text { if } b=0,
\end{array}\right.
$$

(globally asymptotically) stabilizes the control system (2.6) in the sense of $\pi$-trajectories and in the Euler sense.

REMARK 2.8. The feedback given in Theorem 2.7 may not be with values in the control set $U$. However, we can project the values $u(x)$ on the unit ball $\bar{B}$ to get a stabilizing feedback which is locally bounded.

Furthermore, we can exploit the semiconcavity property more strongly to derive some regularity properties on the feedback in the case of affine control systems. We are going to obtain continuity of our discontinuous feedback outside a set of singularity which will be proved small on account of semiconcavity.

THEOREM 2.9. If the control affine system (2.6) is GAC, then there exists a subset $\mathcal{D} \subset \mathbb{R}^{n}$ which verifies the following properties:

(i) The set $\mathcal{D}$ is an open dense set.

(ii) The complement $S$ of $\mathcal{D}$ has Hausdorff dimension no greater than $n-1$.

(iii) There exists a feedback $u: \mathbb{R}^{n} \rightarrow U$ which is continuous on $\mathcal{D}$ for which the closed-loop system (2.6) is globally asymptotically stable in the sense of Carathéodory; in particular, the Euler trajectories are solutions in the sense of Carathéodory.

REMARK 2.10. As in the paper of Artstein [5, Theorem 5.2], if the system verifies the small-control property, then the feedback can be chosen to be continuous at the origin. More precisely, if we assume that there exists a semiconcave control-Lyapunov function such that for all $\epsilon>0$, there exists $\delta>0$ such that $\|x\| \leq \delta$ implies the existence of $u \in U$ with $\|u\|<\epsilon$ and satisfying (2.4), then the feedback given by the previous theorem can be taken to be continuous at the origin. 
We recognize this time in Theorem 2.9 the result of Ancona and Bressan [4] in the case of control affine systems. As in their case, it turns out from the proof that the function $t \mapsto f(x(t), u(x(t))$ is left-continuous. Let us also remark that we get from the upper bound on the Hausdorff dimension of $S$ that this set has Lebesgue measure zero. We refer to Morgan [19] for a survey of the notions of Hausdorff measure and Hausdorff dimension.

Actually, if we consider a control-Lyapunov function given by Theorem 2.4 we will see in the proof of Theorem 2.9 that the construction of the set $\mathcal{D}$ is based on the function

$$
\Psi_{V}(x):=\min _{u \in U} \max _{\zeta \in \partial V(x)}\langle\zeta, f(x, u)\rangle,
$$

where $\partial V$ denotes the Clarke's generalized gradient of $V$; see section 3.1 for the definition. This function is upper semicontinuous on $\mathbb{R}^{n} \backslash\{0\}$; hence if we consider a continuous function $\delta: \mathbb{R}^{n} \rightarrow \mathbb{R}$, the set

$$
\mathcal{D}_{V}^{\delta}:=\left\{x \in \mathbb{R}^{n} \backslash\{0\}: \Psi_{V}(x)<-\delta(x)\right\}
$$

is open. In particular, if the control-Lyapunov function $V$ satisfies an additional assumption concerning the set $\mathcal{D}_{V}^{\delta}$ and the function $\Psi_{V}$, then we can provide a stabilizing feedback which is invariant with respect to $\mathcal{D}_{V}^{\delta}$. Let us state the result.

THEOREM 2.11. Let there be given a GAC control system and a control-Lyapunov function $V$ as in Theorem 2.4. If there exists a continuous and positive definite function

$$
\delta: \mathbb{R}^{n} \rightarrow \mathbb{R}
$$

such that

$$
\forall x \in \mathbb{R}^{n} \backslash\{0\}, \quad \delta(x)<V(x)
$$

and

$$
\forall x \in \mathbb{R}^{n} \backslash\{0\}, \quad \Psi_{V}(x) \leq-\delta(x) \Longrightarrow x \in \mathcal{D}_{V}^{\delta},
$$

then we have the following:

(i) The set $\mathcal{D}_{V}^{\delta}$ is an open dense set and $\mathcal{D}_{V}^{\delta} \cup\{0\}$ is path-connected.

(ii) The complement $S_{V}$ of $\mathcal{D}_{V}^{\delta}$ has Hausdorff dimension no greater than $n-1$.

(iii) There exists a feedback $u: \mathbb{R}^{n} \rightarrow U$ which is smooth on $\mathcal{D}_{V}^{\delta}$ for which the closed-loop system (2.6) is globally asymptotically stable in the sense of Carathéodory. Moreover, for any Carathéodory solution $x(\cdot)$ of this system, we have

$$
x(t) \in \mathcal{D}_{V}^{\delta} \quad \forall t>0 .
$$

In particular, the Euler trajectories are solutions in the sense of Carathéodory.

REMARK 2.12. Let us note that if there exists a positive definite and continuous function $\delta$ such that

$$
\forall x \in \mathbb{R}^{n}, \quad \Psi_{V}(x)<0 \Longrightarrow \Psi_{V}(x) \leq-\delta(x),
$$

then the function $\frac{\delta}{2}$ satisfies (2.8) and (2.9).

We stress that the property (2.10) implies the following facts: For each state $x_{0} \in \mathbb{R}^{n}$, for any Carathéodory solution of the closed-loop system starting at $x_{0}$, the following hold: 
- If $x_{0}=0$, then $x(t)=0$ for all $t \geq 0$.

- If $x_{0} \in \mathcal{D}_{V}^{\delta}$, then $x(t) \in \mathcal{D}_{V}^{\delta} \cup\{0\}$ for all $t \geq 0$; and consequently

$$
\dot{x}(t)=f(x(t), u(x(t))) \quad \forall t \geq 0 \quad \text { such that (s.t.) } x(t) \neq 0 \text {. }
$$

- If $x_{0} \notin \mathcal{D}_{V}^{\delta} \cup\{0\}$, then $x(t) \in \mathcal{D}_{V}^{\delta} \cup\{0\}$ for all $t>0$; and consequently

$$
\dot{x}(t)=f(x(t), u(x(t))) \quad \forall t>0 \quad \text { s.t. } x(t) \neq 0 .
$$

To summarize our results, we have shown that under the additional assumptions (2.8) and (2.9), there exists a feedback which stabilizes our closed-loop system in the sense of Carathéodory, and moreover its Carathéodory trajectories are solutions in the classical sense for positive times whenever $x(t) \neq 0$. Let's also emphasize that the conclusions of Theorem 2.11 imply some topological properties for the set $\mathcal{D}_{V}^{\delta}$. As a matter of fact, the set $\mathcal{D}_{V}^{\delta} \cup\{0\}$ is invariant with respect to a locally Lipschitz vector field (since the functions $f_{0}, f_{1}, \ldots, f_{m}$ are locally Lipschitz), which is asymptotically stabilizing to the origin; therefore it is contractible.

We present in the two following sections two simple examples where the hypotheses (2.8) and (2.9) of Theorem 2.11 are fulfilled.

2.3. One-dimensional systems. Let us assume that the control system is of the form

$$
\dot{x}=u g(x),
$$

where the control $u$ belongs to the interval $[a, b]$ and $g$ is a locally Lipschitz vector field on $\mathbb{R}^{n}$. In this case, the condition (2.9) is always ensured. Let us consider a semiconcave control-Lyapunov $V$ for the system (2.11) and set

$$
\mathcal{D}_{V}:=\left\{x \in \mathbb{R}^{n} \backslash\{0\}: \Psi_{V}(x)<0\right\},
$$

where

$$
\forall x \in R^{n}, \quad \Psi_{V}(x):=\min _{u \in U} \max _{\zeta \in \partial V(x)}\langle\zeta, f(x, u)\rangle .
$$

We have the following.

Lemma 2.13. For any $x \in \mathcal{D}_{V}$,

$$
\Psi_{V}(x) \leq-V(x) .
$$

Proof. Let $x \in \mathcal{D}_{V}$. Thus $\Psi_{V}(x)<0$, and there exists $u \in[a, b]$ such that

$$
\forall \zeta \in \partial V(x), \quad u\langle\zeta, g(x)\rangle<0 .
$$

Without loss of generality we treat the case where $u>0$.

We know by assumption on $V$ that for each $\zeta \in \partial_{L} V(x)$, there exists $u(\zeta) \in[a, b]$ such that

$$
u(\zeta)\langle\zeta, g(x)\rangle \leq-V(x) .
$$

Since $\partial_{L} V(x) \subset \partial V(x)$, we deduce immediately from (2.12) that $u(\zeta)>0$ and that $\Psi(x) \leq-V(x)$.

From this lemma and Remark 2.12 we deduce that the conclusions of Theorem 2.11 apply in the case of one-dimensional systems. We add that we will clearly define the shape of the set of singularities (i.e., $S_{V}$ the complement of $\mathcal{D}_{V}$ ) in the forthcoming paper [22].

REMARK 2.14. Actually, it is not difficult to see that the system (2.11) is GAC and locally stabilizable by a continuous feedback if and only if it is globally stabilizable by a smooth feedback. 
2.4. The nonholonomic integrator. The nonholonomic integrator control system

$$
\begin{aligned}
& \dot{x}_{1}=u_{1}, \\
& \dot{x}_{2}=u_{2}, \\
& \dot{x}_{3}=x_{1} u_{2}-x_{2} u_{1}
\end{aligned}
$$

appeared in [7] as an example of a nonlinear control system which does not satisfy Brockett's condition and cannot be stabilized with continuous feedback. It was shown in [16] that the nonsmooth function

$$
V=\max \left\{\sqrt{x_{1}^{2}+x_{2}^{2}},\left|x_{3}\right|-\sqrt{x_{1}^{2}+x_{2}^{2}}\right\}
$$

is a control-Lyapunov function for the nonholonomic integrator system (2.13). As before,

$$
\Psi_{V}(x):=\min _{u \in U} \max _{\zeta \in \partial V(x)}\left\langle\zeta,\left(u_{1}, u_{2}, x_{1} u_{2}-x_{2} u_{1}\right)^{t}\right\rangle,
$$

and we remark that $\Psi_{V}(x)=0$ on the set

$$
S:=\left\{x \in \mathbb{R}^{n}: x_{1}^{2}+x_{2}^{2}=0\right\} .
$$

In addition, the function $V$ is differentiable outside $S$, and thus by the results given in [16] we get that for any $x \in \mathcal{D}_{V}$,

$$
\Psi_{V}(x) \leq-\frac{V(x)}{\sqrt{4+V(x)^{2}}}=: \delta(x)
$$

Theorem 2.11 and Remark 2.12 now imply the existence of a stabilizing feedback satisfying the properties given in its statement.

2.5. A counterexample. We give in this section for every Euclidean space $\mathbb{R}^{n}$ with $n \geq 2$ an example of a control affine system which is GAC and which does not verify the conditions (2.8) and (2.9) for any control-Lyapunov function $V$ and any continuous positive definite function $\delta$, and for which the conclusions of Theorem 2.11 do not hold.

Let $n \geq 2$ and $x_{0} \in R^{n} \backslash 3 \bar{B}$ be fixed. There exists a locally Lipschitz vector field on $\mathbb{R}^{n}$ such that

$$
f_{0}(x)= \begin{cases}-x & \text { if } x \in \bar{B} \\ x-x_{0} & \text { if } \frac{1}{4} \leq\left\|x-x_{0}\right\| \leq \frac{1}{2} .\end{cases}
$$

Let us also define two auxiliary functions $g_{0}$ and $g_{1}$. We set for any $x \in \mathbb{R}^{n}$,

$$
g_{0}(x):=\max \left\{0,1-d_{K_{1}}(x)\right\},
$$

where $d_{K_{1}}(\cdot)$ denotes the distance function corresponding to the set

$$
K_{1}:=\bar{B} \bigcup\left(x_{0}+\frac{1}{2} \bar{B}\right) \backslash\left(x_{0}+\frac{1}{4} \bar{B}\right) .
$$

Then we set for any $x \in \mathbb{R}^{n}, g_{1}(x):=d_{K_{2}}(x)$ with

$$
K_{2}:=\frac{1}{2} \bar{B} \bigcup \mathcal{A} \subset K_{1},
$$


where $\mathcal{A}$ denotes the annulus $\mathcal{A}=\left(x_{0}+\frac{7}{16} \bar{B}\right) \backslash\left(x_{0}+\frac{5}{16} \bar{B}\right)$.

We now present the dynamics which will form our counterexample; we consider the following control system:

$$
\dot{x}=f(x, u):=g_{0}(x) f_{0}(x)+g_{1}(x) u, \quad(x, u) \in \mathbb{R}^{n} \times \bar{B} .
$$

Let us notice the following facts:

$$
f(x, u)=f_{0}(x) \quad \text { if }(x, u) \in K_{2} \times \bar{B}
$$

and

$$
f(x, u)=g_{1}(x) u \quad \text { if }(x, u) \in\left(\mathbb{R}^{n} \backslash\left(K_{1}+\bar{B}\right)\right) \times \bar{B} .
$$

It is straightforward to show that this affine control system is GAC; let us notice that this is true since we are in a dimension greater than 2. Now, let us assume that there exists an open dense set $\mathcal{D}$ of $\mathbb{R}^{n}$ which is invariant with respect to some smooth (on $\mathcal{D}$ ) stabilizing feedback $k(\cdot)$ and such that its complement $S:=\left(\mathbb{R}^{n} \backslash\{0\}\right) \backslash \mathcal{D}$ is repulsive (see (2.10)).

First, since our dynamics reduce to $f_{0}$ around the origin, we can assume that $0 \in \mathcal{D}$ and hence that $\mathcal{D}$ is contractible. On the other hand, the control system (2.15) coincides with the dynamical system $\dot{x}=f_{0}(x)$ on the interior of $\mathcal{A}$. Consequently, by repulsivity the set $S$ cannot intersect $\operatorname{int}(\mathcal{A})$; hence we deduce that $S$ meets the ball $x_{0}+\frac{5}{16} \bar{B}$. (If the vector field $f(\cdot, k(\cdot))$ were continuous on this ball, it would have an equilibrium on it by Brouwer's theorem; as a matter of fact the ball would be invariant under the dynamic $\dot{x}=-f(x, k(x))$.) In other words, there exists a nonempty compact set $K$ such that

$$
K \subset x_{0}+\frac{3}{8} \bar{B} \subset x_{0}+\frac{7}{16} B
$$

and

$$
K \cap \mathcal{D} \neq \emptyset .
$$

This means that we can write our set $\mathcal{D}$ as follows:

$$
\mathcal{D}=\left(\mathcal{D} \bigcup x_{0}+\frac{7}{16} B\right) \backslash K
$$

In fact, we can see $\mathcal{D}$ as an open set minus a compact subset of itself. Such a set can't be contractible (we refer to algebraic topology for the proof of this result).

In particular, this shows by Theorem 2.11 that the control system defined above does not possess a semiconcave (outside the origin) control-Lyapunov function $V$ (with, for instance, $W=V$ ) and a continuous positive definite function verifying (2.8) and (2.9).

\section{Complementary definitions.}

3.1. Some facts in nonsmooth analysis. We recall briefly some notions of nonsmooth analysis which are essential for this article. We first define $\partial_{P} V(x)$ as the proximal subdifferential of $V$ at $x$ where the function $V: \mathbb{R}^{n} \rightarrow \mathbb{R}$ is assumed to be locally Lipschitz: $\zeta$ belongs to $\partial_{P} V(x)$ if and only if there exists $\sigma$ and $\eta>0$ such that

$$
V(y)-V(x)+\sigma\|y-x\|^{2} \geq\langle\zeta, y-x\rangle \quad \forall y \in x+\eta B .
$$


We further state that this object can be empty at some points. Nevertheless it can be proved that the proximal subdifferential is nonempty on a dense set of $\mathbb{R}^{n}$. Such a property leads us to define the limiting subdifferential and the generalized gradient which will be nonempty at every point. For all $x$ in $\mathbb{R}^{n}$, the limiting subdifferential of $V$ at $x$ is defined as follows:

$$
\partial_{L} V(x):=\left\{\lim \zeta_{k}: x_{k} \rightarrow x, \zeta_{k} \in \partial_{P} V\left(x_{k}\right)\right\} .
$$

REMARK 3.1. Of course, by the construction of the limiting subdifferential and by continuity of $f(\cdot, \cdot)$, the property (2.4) given in Definition 2.3 is equivalent to the following one:

$$
\forall x \in \mathbb{R}^{n} \backslash\{0\}, \forall \zeta \in \partial_{L} V(x), \quad \min _{u \in U}\langle\zeta, f(x, u)\rangle \leq-W(x) .
$$

Finally, we derive the generalized gradient of Clarke as follows:

$$
\partial V(x):=\operatorname{co} \quad \partial_{L} V(x),
$$

where $\operatorname{co} A$ denotes the convex hull of the set $A$.

It is important to note that in our case of a locally Lipschitz function, the definition of the generalized gradient coincides with the following one based on Rademacher's theorem:

$$
\partial V(x):=\operatorname{co}\left\{\lim \nabla V\left(x_{k}\right): x_{k} \rightarrow x, x_{k} \in D_{f} \backslash N\right\},
$$

where $D_{f}$ denotes the set of differentiability of $f$ and $N$ is any set of Lebesgue measure zero in $\mathbb{R}^{n}$.

Moreover, we stress that there exist complete calculi of proximal subdifferentials and generalized gradients, ones that extend all theorems of the usual smooth calculus; our principal references for this theory are the books of Clarke [8] and Clarke et al. [12].

3.2. Results on semiconcave functions. We recall in this subsection some basic properties of the semiconcave functions. Let us first recall this definition; we assume in this section that $\Omega$ is a given open subset of $\mathbb{R}^{n}$.

Definition 3.2. Let $g: \Omega \longrightarrow \mathbb{R}$ be a continuous function on $\Omega$; it is said to be semiconcave on $\Omega$ if for any point $x_{0} \in \Omega$ there exist $\rho, C>0$ such that

$$
g(x)+g(y)-2 g\left(\frac{x+y}{2}\right) \leq C\|x-y\|^{2}
$$

for all $x, y \in x_{0}+\rho B$.

The property (3.6) amounts to the concavity of $x \mapsto g(x)-2 C\|x\|^{2}$, as is easily checked. Hence, a semiconcave function $g$ can be seen locally as the sum of a concave function and a smooth function. In particular, this implies that the semiconcave functions are locally Lipschitz. We know different examples of semiconcave functions. Concave functions are of course semiconcave. Another class of semiconcave functions is that of $C^{1}$ functions with locally Lipschitz gradient. We can in fact give a characterization of the semiconcavity property of a function $g$ by using the proximal superdifferentials defined as follows:

$$
\partial^{P} g(x):=-\partial_{P} g(x)
$$


or, equivalently, $\zeta \in \partial^{P} g(x)$ if and only if there exists $\sigma$ and $\eta>0$ such that

$$
g(y)-g(x)-\sigma\|y-x\|^{2} \leq\langle\zeta, y-x\rangle \quad \forall y \in x+\eta B .
$$

This analytic definition enables us to give a characterization of semiconcavity; we refer to [24] for the proof.

Proposition 3.3. A function $g: \Omega \rightarrow \mathbb{R}$ is semiconcave if and only if $\sigma$ and $\eta$ of (3.8) can be chosen uniform on the compact sets of $\Omega$. Moreover, the superdifferential and the generalized gradients coincide on $\Omega$.

REMARK 3.4. We can in fact relate the semiconcavity property of a given function to some geometric properties of its epigraph; we refer to [24] for such results. Furthermore we can define the semiconcavity property in a more general setting such that semiconcave functions keep the same behavior of concave functions; hence we can relate their differentiability properties to the ones of concave functions (see, for instance, [32]).

Since the semiconcave functions are locally Lipschitz, we get by Rademacher's theorem that they are differentiable almost everywhere. Actually, since they are locally the sum of a semiconcave function and a smooth function, we can state positively by Alexandroff's theorem (see $[2,14]$ ) that the semiconcave functions are twice differentiable almost everywhere.

A study has been devoted to the set of nondifferentiability of such functions. Alberti, Ambrosio, and Cannarsa [1] were able to provide some upper bounds on the dimension of singular sets of semiconcave functions.

Let $g: \Omega \rightarrow \mathbb{R}$ be a semiconcave function. Define

$$
\Sigma^{k}(g):=\{x \in \Omega: \operatorname{dim}(\partial g(x))=k\},
$$

where $k \in[0, n]$ is an integer. Clearly, $\Sigma^{0}(g)$ represents the set of differentiability of $u$, and moreover

$$
\Omega=\bigcup_{k=0}^{n} \Sigma^{k}(g) .
$$

We can evaluate the size of these sets.

Proposition 3.5. For any integer $k \in[0, n]$, the set $\Sigma^{k}(g)$ has Hausdorff dimension $\leq n-k$.

We refer to [1] (see also [3]) for the proof and again to the book of Morgan [19] for a serious survey of the Hausdorff dimension.

Finally, Alberti, Ambrosio, and Cannarsa made some useful links between the Bouligand tangent cones of some subsets of the $\Sigma^{k}(g)$ 's and the generalized gradients of $g$. Let us define for any $\alpha>0$

$$
\Sigma_{\alpha}^{k}(f):=\left\{x \in \Omega: \exists B_{\alpha}^{k} \subset \partial f(x) \text { with } \operatorname{diam}\left(B_{\alpha}^{k}\right)=2 \alpha\right\},
$$

where $B_{\alpha}^{k}$ denotes a ball of dimension $k$ with diameter $\alpha$. For any set $S \subset \mathbb{R}^{n}$, we shall denote by $S^{\perp}$ the set defined as follows:

$$
S^{\perp}:=\left\{p \in \mathbb{R}^{n}: q \mapsto\langle q, p\rangle \text { is constant on } S\right\} .
$$

We have the following result.

Proposition 3.6. The sets $\Sigma_{\alpha}^{k}(g)$ are closed sets, and

$$
T_{\Sigma_{\alpha}^{k}(g)}^{B}(x) \subset[\partial f(x)]^{\perp} \quad \forall x \in \Sigma_{\alpha}^{k}(f) \backslash \Sigma_{\alpha}^{k+1}(f) .
$$

We again refer to the paper of Alberti, Ambrosio, and Cannarsa for the proof. 
3.3. Discontinuous stabilizing feedbacks. As it has been explained before, there do not exist robust stabilizing feedbacks in general. To overcome this difficulty, we describe a concept of solution of the general Cauchy problem

$$
\dot{x}=f(x, u(x)), \quad x(0)=x_{0},
$$

where the feedback $u: \mathbb{R}^{n} \rightarrow U$ is not assumed to be continuous. This concept of solutions for differential equations with discontinuous right-hand side, inspired by the theory of differential games, has been used in the fundamental article of Clarke et al. [10] (see also [9]) to produce discontinuous stabilizing feedbacks; it provides an alternative approach to those developed by Sussmann [31] and Coron [13] (see also Pomet $[20])$.

Let $\pi=\left\{t_{i}\right\}_{i \geq 0}$ be a partition of $[0, \infty)$, by which we mean a countable, strictly increasing sequence $t_{i}$ with $t_{0}=0$ such that $t_{i} \rightarrow \infty$ as $i \rightarrow \infty$. The diameter of $\pi$, denoted $\operatorname{diam}(\pi)$, is defined as $\sup _{i \geq 0}\left(t_{i+1}-t_{i}\right)$. Given an initial condition $x_{0}$, the $\pi$-trajectory $x(\cdot)$ corresponding to $\pi$ is defined in a step-by-step fashion as follows. Between $t_{0}$ and $t_{1}, x(\cdot)$ is a classical solution of the differential equation

$$
\dot{x}(t)=f\left(x(t), u\left(x_{0}\right)\right), \quad x(0)=x_{0}, \quad t_{0} \leq t \leq t_{1} .
$$

(Of course in general we do not have uniqueness of the solution, nor is there necessarily even one solution.) We then set $x_{1}:=x\left(t_{1}\right)$ and restart the system with control value $u\left(x_{1}\right)$ :

$$
\dot{x}(t)=f\left(x(t), u\left(x_{1}\right)\right), \quad x\left(t_{1}\right)=x_{1}, \quad t_{1} \leq t \leq t_{2},
$$

and so on in this fashion. This resulting trajectory $x$ is a physically meaningful one that corresponds to a natural sampling procedure and piecewise constant controls; this kind of solution, called a system sampling solution, is due to Krasovskii and Subbotin (see [15]). We proceed now to give the definition of the global asymptotic stabilization associated to this concept.

DEFINITION 3.7. The system (2.1) is globally asymptotically stable in the sense of $\pi$-trajectories if there exist a function $M: \mathbb{R}_{>0} \longrightarrow \mathbb{R}_{>0}$ such that $\lim _{R \downarrow 0} M(R)=0$ and two functions $T, \delta: \mathbb{R}_{>0} \times \mathbb{R}_{>0} \longrightarrow \mathbb{R}_{\geq 0}$ with the following property:

For any $0<r<R$, for any partition $\pi$ with $\operatorname{diam}(\pi) \leq \delta(r, R)$, and for each initial state $x_{0}$ such that $\left\|x_{0}\right\| \leq R$, the corresponding $\pi$-trajectory $x(\cdot)$ is well-defined and satisfies the following:

(1) for all $t \geq 0,\|x(t)\| \leq M(R)$;

(2) for all $t \geq T(r, R),\|x(t)\| \leq r$.

REMARK 3.8. This definition is equivalent to another one given by Sontag in [27]. In that paper, it was required that there exist a function $\beta \in \mathcal{K} \mathcal{L}$ so that the following property held: For each $0<\epsilon<K$, there exists a $\delta=\delta(\epsilon, K)>0$ such that, for every sampling schedule $\pi$ with $\operatorname{diam}(\pi)<\delta$, and for each initial state $x_{0}$ with $\left\|x_{0}\right\| \leq K$, the corresponding $\pi$-trajectory $x(\cdot)$ of (2.1) is well-defined and satisfies

$$
\left\|x_{\pi}(t)\right\| \leq \max \{\beta(K, t), \epsilon\} \quad \forall t \geq 0 .
$$

We can define from the concept of $\pi$-trajectories the notion of Euler trajectories. As presented in [25], we call an Euler solution of (2.2) any uniform limit of $\pi$-trajectories of this system with $\operatorname{diam}(\pi) \rightarrow 0$. Moreover, we will say that the closed-loop system (2.2) is globally asymptotically stable in the Euler sense (or that 
the feedback $u$ stabilizes in the Euler sense) if the two properties given in Definition 2.1 are satisfied for any Euler solutions.

We also recall briefly for the convenience of the reader that a function $x(\cdot)$ : $[0, \infty) \rightarrow \mathbb{R}^{n}$ is called a Carathéodory solution (or trajectory) of our closed-loop system if it satisfies

$$
\dot{x}(t)=f(x(t), u(x(t)) \quad \text { a.e. } \forall t \geq 0 .
$$

We will say in that case that the closed-loop system is stabilizing in the sense of Carathéodory.

4. Proof of Theorem 2.4. We can invoke the main result of [23] to get a control-Lyapunov function $V_{0}$ which is semiconcave on $\mathbb{R}^{n} \backslash\{0\}$. We begin by showing that there exists a smooth function $\gamma:(0, \infty) \rightarrow(0, \infty)$ which satisfies

$$
\min _{u \in U}\langle\zeta, f(x, u)\rangle \leq-\gamma\left(V_{0}(x)\right) \quad \forall x \in \mathbb{R}^{n} \backslash\{0\}, \forall \zeta \in \partial_{P} V_{0}(x) .
$$

We use the method given by Clarke, Ledyaev, and Stern in [11]. We set for all $v>0$,

$$
\gamma(v):=\min \{W(x) ; x \in \Gamma(v)\}
$$

where

$$
\Gamma(v):=\left\{x \in \mathbb{R}^{n} ; V_{0}(x)=v\right\} .
$$

It is not difficult to show that the multifunction $\Gamma$ is locally Lipschitz, which implies that the function $\gamma$ is locally Lipschitz on $(0, \infty)$ and verifies (4.1). Moreover, we can approximate $\gamma$ by a smooth function $\tilde{\gamma}$ such that

$$
0<\tilde{\gamma} \leq \gamma .
$$

Finally, without loss of generality we can suppose that $\gamma$ is smooth and verifies (4.1). Now, we set

$$
\Psi(t):=\int_{1}^{t} \frac{1}{\gamma(s)} d s
$$

This new function from $(0, \infty)$ into $\mathbb{R}$ is increasing, smooth, and verifies the three following properties:

$$
\begin{gathered}
\Psi^{\prime}(t)=\frac{1}{\gamma(t)} \quad \forall t>0, \\
\limsup \Psi(t) \leq 0,
\end{gathered}
$$

and

$$
\liminf _{t \rightarrow \infty} \Psi(t) \geq 0
$$

We are now able to define a new control-Lyapunov function $V_{1}$. We set

$$
V_{1}(x):= \begin{cases}V_{0}(x) e^{c \Psi\left(V_{0}(x)\right)} & \text { if } x \neq 0 \\ 0 & \text { if } x=0\end{cases}
$$


By (4.4) and (4.5) and the properties of $V_{0}$, this new function is obviously proper, continuous at the origin, and locally Lipschitz on $\mathbb{R}^{n} \backslash\{0\}$. We now want to make the link between the proximal subdifferentials of $V_{1}$ and the proximal subdifferentials of $V_{0}$; for that, we give the following lemma.

LEMma 4.1. Let there be given two functions $f: \Omega \rightarrow \mathbb{R}$ and $F: \mathbb{R} \rightarrow \mathbb{R}$. If we assume that $f$ is positive and locally Lipschitz on the open set $\Omega$ and that $F$ is a $C^{2}$, positive, and increasing $\left(F^{\prime}>0\right)$ function, then for all $x \in \Omega$,

$$
\partial_{P}[f F(f)](x)=\left[F(f(x))+f(x) F^{\prime}(f(x))\right] \partial_{P} f(x) .
$$

The same formula holds for the proximal superdifferential. Moreover, if the function $f$ is taken to be semiconcave, then the new function $f F(f)$ is semiconcave as well.

Proof. Let us consider $x \in \mathbb{R}^{n}$ and $\zeta \in \partial_{P}[f F(f)](x)$; then by (3.1), there exists $\sigma \geq 0$ such that

$$
f(y) F(f(y))-f(x) F(f(x))+\sigma\|y-x\|^{2} \geq\langle\zeta, y-x\rangle
$$

whenever $y$ is in a neighborhood of $x$. The function $X \rightarrow X F(X)$ is $C^{2}$, so we have by Taylor's formula that there exists a constant $C$ such that for all $Y$ in a neighborhood of $X$,

$$
Y F(Y)-X F(X)=F(X)+X F^{\prime}(X)(Y-X)+\frac{C}{2}\|Y-X\|^{2}+o\left(\|Y-X\|^{2}\right) .
$$

We get for $Y=f(y)$ and $X=f(x)$ that $f(y) F(f(y))-f(x) F(f(x))$ is equal to

$$
\left[F(f(x))+f(x) F^{\prime}(f(x))\right][f(y)-f(x)]+\frac{C}{2}\|f(y)-f(x)\|^{2}+h,
$$

where $h=o\left(\|f(y)-f(x)\|^{2}\right)$.

We set $D:=F(f(x))+f(x) F^{\prime}(f(x))$; by the assumptions on $f$ and $F, D>0$, and so we can divide by $D$. On the other hand, $f$ being locally Lipschitz, we deduce that there exists a constant $\bar{\sigma} \geq 0$ such that

$$
\frac{f(y)}{D}-\frac{f(x)}{D}+\bar{\sigma}\|y-x\|^{2} \leq\left\langle\frac{\zeta}{D}, y-x\right\rangle
$$

whenever $y$ is in a neighborhood of $x$; and then by the characterization (3.1) we get

$$
\partial_{P} f[F(f)](x) \subset\left[F(f(x))+f(x) F^{\prime}(f(x))\right] \partial_{P} f(x) .
$$

This proves one inclusion; the other is left to the reader. Of course, for the case of the proximal superdifferential, a similar proof is valid.

It remains to show the conservation of semiconcavity. If we assume that $f$ is semiconcave, then by using Proposition 3.3 and following the same proof as above, we show that the different $\sigma$ remain uniform on the compact sets of $\Omega$.

We now turn back to the proof of Theorem 2.4; the lemma implies immediately that for all $x \in \mathbb{R}^{n} \backslash\{0\}$ and all $\zeta \in \partial_{P} V_{1}(x) \subset \partial_{L} V_{1}(x)$,

$$
\begin{aligned}
\min _{u \in U}\langle\zeta, f(x, u)\rangle & \leq-\gamma\left(V_{0}(x)\right)\left[e^{\Psi\left(V_{0}(x)\right)}+c \Psi^{\prime}\left(V_{0}(x)\right) V_{0}(x) e^{\Psi\left(V_{0}(x)\right)}\right] \\
& \leq-V_{1}(x)\left[\frac{\gamma\left(V_{0}(x)\right)}{V_{0}(x)}+c \gamma\left(V_{0}(x)\right) \psi^{\prime}\left(V_{0}(x)\right)\right] \\
& \leq-V_{1}(x)\left[\frac{\gamma\left(V_{0}(x)\right)}{V_{0}(x)}+c\right] \quad \text { by }(4.3) \\
& \leq-c V_{1}(x) .
\end{aligned}
$$


On the other hand, as the initial function $V_{0}$ was semiconcave on $\mathbb{R}^{n} \backslash\{0\}$, we have by Lemma 4.1 that the new function $V_{1}$ is semiconcave; the proof of Theorem 2.4 is complete.

5. Proof of Theorems 2.5 and 2.7. We will treat only the case where the control-Lyapunov function is that given by Theorem 2.4. The general case of a controlLyapunov function related to a function $W$ is left to the reader.

Let $V$ be the semiconcave Lyapunov function given by Theorem 2.4 and two positive constants $r<R$. We set

$$
M_{R}:=\max \{V(x):\|x\| \leq R\} \quad \text { and } \quad M(R):=\max \left\{\|y\|: V(y) \leq M_{R}\right\} .
$$

Obviously, the function $M(\cdot)$ is nondecreasing and verifies

$$
\lim _{R \downarrow 0} M(R)=0 .
$$

We also set two constants depending on $r$ :

$$
m_{r}:=\min \{V(x):\|x\| \geq r\} \quad \text { and } \quad m_{\frac{r}{2}}:=\min \left\{V(x):\|x\| \geq \frac{r}{2}\right\} ;
$$

we can say by definition that if $V(x) \leq \frac{m_{\frac{r}{2}}}{2}$, then $x \in \frac{r}{2} \bar{B}$. On the other hand, the Proposition 3.3 allows us to consider $\sigma:=\sigma\left(\frac{r}{2}, R\right)$ and $\delta$ uniform on the set $\mathcal{A}:=\left\{x: \frac{m_{\frac{r}{2}}}{2} \leq V(x) \leq M_{R}\right\} \subset \mathbb{R}^{n} \backslash\{0\}$.

We get that for all $x \in \mathcal{A}$, all $y \in \mathcal{A}$, and all $\zeta \in \partial_{L} V(x)$,

$$
-V(y)+V(x)+\sigma\|y-x\|^{2} \geq\langle-\zeta, y-x\rangle .
$$

From now on, we denote by $M_{f}$ the upper bound of $f$ on $R \bar{B} \times U$, by $L_{f}$ the Lipschitz constant of $f$ on the same set, by $m_{V}$ the minimum of $V$, and by $L_{V}$ the Lipschitz constant of $V$ on $\mathcal{A}$. Let us consider a $\pi$-trajectory $x(\cdot)$ associated to a partition $\pi=\left\{0=t_{0}<t_{1}<\cdots\right\}$ and to nodes $x_{i}:=x\left(t_{i}\right)$ with $x_{0} \in \mathcal{A}$. We pick $\zeta_{0}$ belonging to $\partial_{L} V\left(x_{0}\right)$. For any $t \in\left[t_{0}, t_{1}\right]$, we can compute by (5.1)

$$
\begin{aligned}
V(x(t))-V\left(x_{0}\right) \leq & \left\langle\zeta_{0}, x(t)-x_{0}\right\rangle+\sigma\left\|x(t)-x_{0}\right\|^{2} \\
\leq & \left\langle\zeta_{0}, \int_{t_{0}}^{t} f\left(x(s), u\left(x_{0}\right)\right) d s\right\rangle+\sigma\left\|x(t)-x_{0}\right\|^{2} \\
\leq & \left\langle\zeta_{0},\left(t-t_{0}\right) f\left(x_{0}, u\left(x_{0}\right)\right)\right\rangle \cdots \\
& +\left\langle\zeta_{0}, \int_{t_{0}}^{t}\left[f\left(x(s), u\left(x_{0}\right)\right)-f\left(x_{0}, u\left(x_{0}\right)\right)\right] d s\right\rangle+\sigma\left\|x(t)-x_{0}\right\|^{2} \\
\leq & -\left(t-t_{0}\right) V\left(x_{0}\right)+\left\|\zeta_{0}\right\| L_{f} \max _{s \in\left[t_{0}, t_{1}\right]}\left\|x(s)-x_{0}\right\|\left(t-t_{0}\right) \cdots \\
& +\sigma\left\|x(t)-x_{0}\right\|^{2} \\
\leq & -\left(t-t_{0}\right) V\left(x_{0}\right)+L_{V} L_{f} M_{f}\left(t-t_{0}\right)^{2}+\sigma M_{f}^{2}\left(t-t_{0}\right)^{2} \\
\leq & \left(t-t_{0}\right)\left[-m_{V}+\left(L_{f} L_{V} M_{f}+\sigma M_{f}^{2}\right)\left(t-t_{0}\right)\right] .
\end{aligned}
$$

More generally, we have for all $t \in\left[t_{i}, t_{i+1}\right]$,

$$
V(x(t))-V\left(x_{i}\right) \leq-\left(t-t_{i}\right) V\left(x_{i}\right)+\left[L_{V} L_{f} M_{f}+\sigma M_{f}^{2}\right]\left(t-t_{i}\right)^{2} .
$$


We get that for any $n$ and for all $t \in\left[t_{n-1}, t_{n}\right]$,

$$
\begin{aligned}
V(x(t))-V\left(x_{0}\right) \leq & \sum_{i=0}^{n-2}\left[-\left(t_{i+1}-t_{i}\right) V\left(x_{i}\right)+\left(L_{V} L_{f} M_{f}+\sigma M_{f}^{2}\right)\left(t_{i+1}-t_{i}\right)^{2}\right] \cdots \\
& -\left(t-t_{n-1}\right) V\left(t_{n-1}\right)+\left(L_{f} L_{V} M_{f}+\sigma M_{f}^{2}\right)\left(t-t_{n-1}\right)^{2} \\
\leq & \left(t-t_{0}\right)\left[-m_{V}+\left(L_{f} L_{V} M_{f}+\sigma M_{f}^{2}\right) \operatorname{diam}(\pi)\right] .
\end{aligned}
$$

We deduce that if we set

$$
\delta(r, R):=\min \left\{\frac{m_{V}}{2\left(L_{f} L_{V} M_{f}+\sigma M_{f}^{2}\right)}, \frac{m_{r}-m_{\frac{r}{2}}}{2 L_{V} M_{f}}\right\}
$$

we obtain from (5.3) that for every $\pi$-trajectory $x(\cdot)$ starting at $x_{0}$ and such that $\operatorname{diam}(\pi) \leq \delta(r, R)$, we have

$$
\forall t \geq 0, \quad V(x(t))-V\left(x_{0}\right) \leq-\frac{m_{V}}{2}\left(t-t_{0}\right) .
$$

That means that the $\pi$-trajectory remains in $\left\{x: V(x) \leq V\left(x_{0}\right)\right\}$, which is included in $M(R) \bar{B}$, and that for $t \geq T(r, R):=\frac{2 M_{R}-m_{\frac{r}{2}}}{m_{V}}$,

$$
V(x(t)) \leq V\left(x_{0}\right)-\frac{m_{V}}{2} t \leq \frac{m_{\frac{r}{2}}}{2}
$$

that is, $x(t) \in \frac{r}{2} \bar{B}$. There is a possible danger! The work done above is valid only when we stay in the set $\mathcal{A}$. But as $\delta(r, R) \leq \frac{m_{r}-m_{\frac{r}{2}}}{2 L_{V} M_{f}}$, there exists a first step $i_{0}$ for which $\frac{m_{\frac{r}{2}}}{2} \leq V\left(x_{i_{0}}\right) \leq \frac{m_{r}}{2}$, and by the same computation as above, the set $\left\{x: V(x) \leq V\left(x_{i_{0}}\right)\right\}$ is invariant, that is,

$$
\forall t \geq t_{i_{0}}, \quad x(t) \in\left\{x: V(x) \leq V\left(x_{i_{0}}\right)\right\} \subset r \bar{B} .
$$

This completes the proof for the case of $\pi$-trajectories.

We get from this proof (more especially from (5.2) and a convergence result of a Riemann's sums) that for any Euler trajectory of (2.2), we have that

$$
V(x(t))-V(x(s)) \leq-\int_{s}^{t} V(x(y)) d y \quad \forall 0 \leq s \leq t .
$$

Gronwall's lemma now brings a proof of the property (2.4).

We now make the proof of Theorem 2.7.

Proof. As in the statement of Theorem 2.5, the formula given above considers for all $x$ a limiting subgradient $\zeta_{V}(x)$ and a function $u(\cdot)$ satisfying

$$
\left\langle\zeta_{V}(x), f(x, u(x))\right\rangle \leq-V(x) \quad \forall x \in \mathbb{R}^{n} \backslash\{0\} .
$$

This construction agrees with the one given in the statement of Theorem 2.5; the result follows. 
6. Proof of Theorem 2.9. Theorem 2.9 requires a more subtle proof; we will need the following lemma and refer to the book of Clarke et al. [12] or to [24] for the proof.

LEMma 6.1. Let $f: \mathbb{R}^{n} \rightarrow \mathbb{R}$ be a locally Lipschitz function and $x \in \mathbb{R}^{n}$; if $\partial_{P} f(x)$ and $\partial^{P} f(x)$ are nonempty, then

$$
\partial_{P} f(x)=\partial^{P} f(x)=\partial f(x)=\{\nabla f(x)\} .
$$

We know by Theorem 2.4 and Remark 3.1 that

$$
\forall x \in \mathbb{R}^{n} \backslash\{0\}, \quad \max _{\zeta \in \partial_{L} V(x)} \min _{u \in U}\langle\zeta, f(x, u)\rangle \leq-V(x)<0 .
$$

We set the function $\Psi: \mathbb{R}^{n} \longrightarrow \mathbb{R}$ as follows:

$$
\forall x \in \mathbb{R}^{n}, \quad \Psi(x):=\min _{u \in U} \max _{\zeta \in \partial V(x)}\langle\zeta, f(x, u)\rangle .
$$

LEMMA 6.2. The function $\Psi$ is upper semicontinuous.

Proof. Since the function $\zeta \mapsto\langle\zeta, f(x, u)\rangle$ is upper continuous and the function $f$ is continuous, we deduce that the function

$$
x \mapsto \max _{\zeta \in \partial V(x)}\langle\zeta, f(x, u)\rangle
$$

is upper semicontinuous. To conclude, we know that a minimum of upper semicontinuous functions is upper semicontinuous.

We define now the following sets:

$$
\mathcal{D}:=\left\{x \in \mathbb{R}^{n} \backslash\{0\} \text { s.t. } \Psi(x)<-\frac{V(x)}{2}\right\} \quad \text { and } \quad S=\mathbb{R}^{n} \backslash \mathcal{D} .
$$

Lemma 6.3. The set $\mathcal{D}$ is an open dense set of $\mathbb{R}^{n}$ and

$$
\mathcal{H}-\operatorname{dim} S \leq n-1 .
$$

Proof. Since the multivalued mapping $x \mapsto \partial V(x)$ has a closed graph, the set $\mathcal{D}$ is obviously open. On the other hand, by the density theorem [12, Theorem 3.1], the proximal subdifferential $\partial_{P} V(x)$ is nonempty on a dense set. Consequently, by the semiconcavity of $V$, both proximal sub- and superdifferentials are nonempty on this set; it implies that (by Lemma 6.1) $\partial_{P} V(x)=\partial_{L} V(x)=\{\nabla V(x)\}$ on a dense subset of $\mathbb{R}^{n}$. So, we conclude that the min-max and the max-min of (6.1) and of the definition of the set $\mathcal{D}$ coincide on an open dense set of $\mathbb{R}^{n}$; that means that $\mathcal{D}$ contains this set. Consequently $\mathcal{D}$ is an open dense set of $\mathbb{R}^{n}$. Moreover, the complement $S$ of $\mathcal{D}$ is included in $\cup_{k=1, \ldots, n} S^{k}(V)$; therefore we get the upper bound on the Hausdorff dimension of $S$ by Proposition 3.5 given in section 3.2.

We define the following multifunction on $\mathcal{D}$ :

$$
\forall x \in \mathcal{D}, \quad G(x):=\left\{u \in U: \forall \zeta \in \partial V(x),\langle\zeta, f(x, u)\rangle \leq-\frac{V(x)}{2}\right\} .
$$

Lemma 6.4. The multifunction $G$ has nonempty closed convex values and is lower semicontinuous on $\mathcal{D}$.

Proof. Since the system is affine in the control, the multifunction $G$ has nonempty closed convex values. We show now that $G$ is lower semicontinuous on $\mathcal{D}$ (we refer to 
[6] for a task about semicontinuity of multivalued functions). We then have to prove that for any sequence $\left(x_{n}\right)_{n}$ of points in $\mathcal{D}$ converging to $x \in \mathcal{D}$, and for any $z \in G(x)$, there exists a sequence $\left(z_{n}\right)_{n}$ of points in $G\left(x_{n}\right)$ with limit $z$.

Let $\left(x_{n}\right)_{n}$ be a sequence in $\mathcal{D}$ converging to $x \in \mathcal{D}$, and let $y=f\left(x, u_{0}\right)$ in $G(x)$. We set, for all $n$,

$$
z_{n}:=f\left(x_{n}, u_{0}\right)
$$

From now on, we denote by $M_{1}$ the Lipschitz constant of $V$, by $M_{2}$ the upper bound of $f\left(\cdot, u_{0}\right)$, and by $M_{3}$ the Lipschitz constant of $f\left(\cdot, u_{0}\right)$ in a neighborhood of $x$ containing all the $x_{n}$ (without loss of generality we can assume this condition), and on the other hand we denote by $\beta(A, B)$ the Hausdorff distance between the sets $A$ and $B$ (see $[6])$. Two cases appear.

1. $\max _{\zeta \in \partial V(x)}\langle\zeta, y\rangle<-\frac{V(x)}{2}$.

We fix $n$ and we choose $\zeta_{n} \in \partial V\left(x_{n}\right)$; so we have

$$
\begin{aligned}
\left\langle\zeta_{n}, z_{n}\right\rangle & =\left\langle\zeta, z_{n}\right\rangle+\left\langle\zeta_{n}-\zeta, z_{n}\right\rangle \quad\left(\text { where } \zeta:=\operatorname{proj}_{\partial V(x)}\left(\zeta_{n}\right)\right) \\
& \leq\langle\zeta, y\rangle+M_{1}\left\|z_{n}-y\right\|+M_{2} \beta\left(\partial V\left(x_{n}\right), \partial V(x)\right) \\
& <-\frac{V(x)}{2}+M_{1}\left\|z_{n}-y\right\|+M_{2} \beta\left(\partial V\left(x_{n}\right), \partial V(x)\right) \\
& <-\frac{V\left(x_{n}\right)}{2}+\frac{M_{3}}{2}\left\|x_{n}-x\right\|+M_{3}\left\|x_{n}-x\right\|+M_{2} \beta\left(\partial V\left(x_{n}\right), \partial V(x)\right) .
\end{aligned}
$$

Hence, for $n$ sufficiently high, $z_{n} \in G\left(x_{n}\right)$, and the sequence $\left(z_{n}\right)_{n}$ converges to $y=f\left(x, u_{0}\right)$ by continuity of $f$.

2. $\max _{\zeta \in \partial V(x)}\langle\zeta, y\rangle=-\frac{V(x)}{2}$.

We know by assumption that since $x \in \mathcal{D}$ there exists $u_{1} \in U$ such that

$$
\forall \zeta \in \partial V(x), \quad\left\langle\zeta, f\left(x, u_{1}\right)\right\rangle<-\frac{V(x)}{2} .
$$

Consequently, we can express $y=f\left(x, u_{0}\right)$ as a limit of

$$
y_{p}=t_{p} y+\left(1-t_{p}\right) f\left(x, u_{1}\right)
$$

(when $t_{p} \uparrow 1$ ) such that $\max _{\zeta \in \partial V(x)}\left\langle\zeta, y_{p}\right\rangle<-\frac{V(x)}{2}$. On the other hand, by the first case each $y_{p}$ is the limit of some sequence $\left(z_{p}^{n}\right)_{n}$; we conclude by a diagonal process.

We conclude that $G$ is a lower semicontinuous multifunction on the set $\mathcal{D}$.

Returning to the proof of our theorem, we can apply the well-known selection theorem of Michael $[18,6]$ to deduce the existence of a continuous selection $u$ of $G$ on $\mathcal{D}$.

Let us now set $\mathcal{E}$ as the set defined by

$$
\mathcal{E}:=\left\{x \in \mathbb{R}^{n} \backslash\{0\} \text { s.t. } \Psi(x)=-\frac{V(x)}{2}\right\} .
$$

Lemma 6.5. For each $x$ in $\mathcal{E}$, there exists a unique $f(x, u) \in U$ such that

$$
\max _{\zeta \in \partial_{C} V(x)}\langle\zeta, f(x, u)\rangle=-\frac{V(x)}{2} .
$$


Proof. This is due to the assumption of strict convexity on the set of control $U$. If $f_{0}(x) \neq 0$ we leave it to the reader to prove that, modifying the dynamics if necessary, the lemma holds.

We are now able to complete the construction of our feedback $u(\cdot)$. We set for each $x \in \mathcal{E}, u(x):=u$, where $u$ is the $u$ of Lemma 6.5. Moreover, for each $x \in S \backslash \mathcal{E} \backslash\{0\}$, we set $u(x):=u$, where $u$ verifies

$$
\exists \zeta \in \partial_{L} V(x), \quad\langle\zeta, f(x, u)\rangle \leq-V(x) .
$$

We have defined our feedback on all the space (of course, we set $u(0)=0$ ). Let us now prove the rest of the theorem. Consider the closed-loop system

$$
\dot{x}=f_{0}(x)+\sum_{i=1}^{m} u_{i}(x) f_{i}(x)
$$

and show that it is globally asymptotically stable with respect to the Carathéodory solutions. Let us first show that the property (2.10) holds for Euler trajectories.

Lemma 6.6. For any Euler trajectory $x(\cdot)$ of $(6.2)$, we have

$$
\Psi(x(t)) \leq-\frac{V(x(t))}{2} \quad \forall t>0 \text { s.t. } x(t) \neq 0 .
$$

Moreover

$$
\dot{x}(t)=f(x(t), u(x(t))) \quad \text { a.e. } t>0 .
$$

Proof. Let us consider $x_{0} \in \mathbb{R}^{n} \backslash\{0\}$ and $x(\cdot)$ is an Euler trajectory of (6.2) with $x(0)=x_{0}$. Obviously, if $x_{0}=0$, then since $f(0, u(0))=0$ all the Euler solutions of (6.2) starting at $x_{0}=0$ will stay at the origin; then the property (6.4) holds. Let us now assume that $x_{0} \neq 0$.

Let $t_{0}$ be fixed in $(0, \infty)$; there exists $\sigma>0$ such that for any $\zeta \in \partial^{P} V\left(x\left(t_{0}\right)\right)$, we have that

$$
-V(y)+V\left(x\left(t_{0}\right)\right)+\sigma\left\|y-x\left(t_{0}\right)\right\|^{2} \geq\left\langle-\zeta, y-x\left(t_{0}\right)\right\rangle
$$

whenever $y$ is in a neighborhood of $x\left(t_{0}\right)$. We deduce that for some $s<t_{0}$ and close to $t_{0}$, we have

$$
V\left(x\left(t_{0}\right)\right)-V(x(s))+\sigma\left\|x(s)-x\left(t_{0}\right)\right\|^{2} \geq\left\langle\zeta, x\left(t_{0}\right)-x(s)\right\rangle .
$$

This implies

$$
\begin{aligned}
\left\langle\zeta, x\left(t_{0}\right)-x(s)\right\rangle & \leq V\left(x\left(t_{0}\right)\right)-V(x(s))+\sigma\left\|x(s)-x\left(t_{0}\right)\right\|^{2} \\
& \leq-\int_{s}^{t_{0}} \frac{V(x(y))}{2} d y+\sigma\left\|x(s)-x\left(t_{0}\right)\right\|^{2} \quad \text { by }(5.5) .
\end{aligned}
$$

Now, by convexity of $f(x(t), U)$ (since $f$ is affine in the control) there exists a sequence $\left(s_{n}\right)_{n}$ and $u_{0}$ in $U$ such that

$$
\lim _{n \rightarrow \infty} \frac{x\left(t_{0}\right)-x\left(s_{n}\right)}{t_{0}-s_{n}}=f\left(x\left(t_{0}\right), u_{0}\right) .
$$


Consequently, passing to the limit for the sequence $\left(s_{n}\right)_{n}$, we obtain

$$
\left\langle\zeta, f\left(x\left(t_{0}\right), u_{0}\right)\right\rangle \leq-\frac{V\left(x\left(t_{0}\right)\right)}{2} .
$$

We can repeat this argument for all $\zeta \in \partial^{P} V\left(x\left(t_{0}\right)\right)$, that is,

$$
\forall \zeta \in \partial^{P} V\left(x\left(t_{0}\right)\right), \quad\left\langle\zeta, f\left(x\left(t_{0}\right), u_{0}\right)\right\rangle \leq-\frac{V\left(x\left(t_{0}\right)\right)}{2} .
$$

Since $\partial^{P} V\left(x\left(t_{0}\right)=\partial V\left(x\left(t_{0}\right)\right)\right.$, that means that

$$
\Psi\left(x\left(t_{0}\right)\right) \leq-\frac{V\left(x\left(t_{0}\right)\right)}{2} .
$$

Hence, we deduce that for any $t>0,(6.3)$ is satisfied and

$$
x(t) \in \mathcal{D} \cup \mathcal{E} .
$$

Two cases appear. If $x(t) \in \mathcal{D}$, then by continuity of $u(\cdot)$ in a neighborhood of $x(t)$, we have $\dot{x}(t)=f(x(t)), u(x(t))$.

Otherwise, $x(t) \in \mathcal{E}$. In this case, Lemma 6.5 asserts that the set of limits of the form (6.6) is a singleton. Thus, we deduce that the function $x(\cdot)$ is left-derivable on $(0, \infty)$ with derivate $f(x(t), u(x(t)))$.

Now, since the trajectory $x(\cdot)$ is locally Lipschitz on $[0, \infty)$, Rademacher's theorem asserts that it is derivable almost everywhere. Then we conclude that this derivate coincides with $f(x(t), u(x(t)))$ almost everywhere; consequently, the Euler trajectories are solutions in the sense of Carathéodory.

Consider now the case of solutions in the sense of Carathéodory. Let $x_{0} \neq 0$ and let $x(\cdot)$ be a Carathéodory solution of (6.2) starting at $x_{0}$. Hence, we have a set $N$ of measure zero on $[0, \infty)$ such that

$$
\dot{x}(t)=f(x(t), u(x(t))) \quad \forall t \in[0, \infty) \backslash N .
$$

We have by the mean value inequality (see [12, Exercise 2.7(d), p. 122]) that for any $0 \leq t<t^{\prime}$, there exists $z_{t, t^{\prime}} \in\left[x(t), x\left(t^{\prime}\right)\right]$ and $\zeta_{t, t^{\prime}} \in \partial_{L} V\left(z_{t, t^{\prime}}\right)$ such that

$$
V\left(x\left(t^{\prime}\right)\right)-V(x(t)) \leq\left\langle\zeta_{t, t^{\prime}}, x\left(t^{\prime}\right)-x(t)\right\rangle .
$$

Now by setting the function $\theta: \mathbb{R}_{\geq 0} \rightarrow \mathbb{R}, \theta(t):=V(x(t))$, it means that for any $t, t^{\prime} \geq 0$

$$
\theta\left(t^{\prime}\right)-\theta(t) \leq\left\langle\zeta_{t, t^{\prime}}, x\left(t^{\prime}\right)-x(t)\right\rangle .
$$

Since the function $f$ is locally bounded, the function $\theta$ is locally Lipschitz and hence by Rademacher's theorem differentiable outside a set of measure zero $N^{\prime}$. Therefore, for all $t \in[0, \infty) \backslash N \cup N^{\prime}$, we obtain by passing to the limit in (6.9)

$$
\theta^{\prime}(t) \leq\langle\zeta, \dot{x}(t)\rangle=\langle\zeta, f(x(t), u(x(t)))\rangle
$$

where $\zeta \in \partial_{L} V(x(t))$.

Lemma 6.7. The Carathéodory trajectory $x(\cdot)$ does not belong to $S \backslash \mathcal{E}$ almost everywhere:

$$
x(t) \notin S \backslash \mathcal{E} \quad \text { a.e. } t \geq 0 .
$$


The proof is based on the properties on the sets $S^{k}(V)$ given in section 3.2 and is postponed to the end of this section.

By Lemma 6.7 there exists a third set $N^{0}$ of measure zero such that

$$
x(t) \in \mathcal{D} \quad \forall t \in[0, \infty) \backslash N^{0} .
$$

Thus we get by construction of $u$ that for all $t \in[0, \infty) \backslash N \cup N^{\prime} \cup N^{0}$,

$$
\theta^{\prime}(t) \leq-\frac{V(x(t))}{2}
$$

Therefore, we deduce by the characterization given in section 3.1 that for any $t \geq 0$

$$
\partial \theta(t) \subset\left(-\infty,-\frac{V(x(t))}{2}\right] .
$$

We deduce that the function $t \mapsto \theta(t)+\int_{0}^{t} \frac{V(x(s))}{2} d s$ is nonincreasing, and consequently

$$
\forall 0 \leq s \leq t, \quad V(x(t))-V(x(s)) \leq-\int_{s}^{t} \frac{V(x(y))}{2} d y .
$$

Now if we fix $t_{0}>0$, and if we take $\zeta \in \partial^{P} V\left(x\left(t_{0}\right)\right)$, we get by (6.5) and (6.10) that

$$
\left\langle\zeta, x\left(t_{0}\right)-x(s)\right\rangle \leq-\int_{s}^{t_{0}} \frac{V(x(y))}{2} d y+\sigma\left\|x(s)-x\left(t_{0}\right)\right\|^{2} .
$$

Then we deduce as in the case of Euler trajectory that for all $t>0$,

$$
x(t) \in \mathcal{D} \cup \mathcal{E} .
$$

Now, Gronwall's lemma easily gives

$$
\forall t>0, \quad V(x(t)) \leq e^{-\frac{t}{2}} v\left(x_{0}\right)
$$

for any Euler trajectory and any Carathéodory trajectory starting at $x_{0}$. We get that the closed-loop system (6.2) is globally asymptotically stable.

It remains to prove Lemma 6.7 .

Proof. Assume that the conclusion is false. Then there would exist a subset $H$ of $[0, \infty)$ of positive measure such that $x(\cdot)$ is differentiable in $H$ and

$$
x(t) \in S \backslash \mathcal{E} \quad \forall t \in H .
$$

On the other hand by (3.9), we can write

$$
\begin{aligned}
S \backslash \mathcal{E} & =\bigcup_{k=1}^{n} \Sigma^{k}(V) \cap S \backslash \mathcal{E} \\
& =\bigcup_{k=1}^{n} \bigcup_{p \in\{1,2, \ldots\}} \Sigma_{\frac{1}{p}}^{k}(V) \cap S \backslash \mathcal{E} .
\end{aligned}
$$

Thus there exists a couple $(k, p)$ for which

$$
x(t) \in \Sigma_{\frac{1}{p}}^{k}(V) \cap S \backslash \mathcal{E} \subset \Sigma_{\frac{1}{p}}^{k}(V)
$$


on a set of positive measure $H^{\prime} \subset H$. This implies that there exists a $t_{0} \in H^{\prime}$ such that

$$
\dot{x}\left(t_{0}\right)=f\left(x\left(t_{0}\right), u\left(x\left(t_{0}\right)\right)\right) \in T_{\Sigma_{\frac{1}{p}}^{k}(V)}^{B}\left(x\left(t_{0}\right)\right) .
$$

Hence we deduce by Proposition 3.6 that

$$
\forall \zeta \in \partial V(x), \quad\left\langle\zeta, f\left(x\left(t_{0}\right), u\left(x\left(t_{0}\right)\right)\right)\right\rangle=-V\left(x\left(t_{0}\right)\right) .
$$

This last inequality implies that $x\left(t_{0}\right) \in \mathcal{D}$; we get a contradiction.

7. Proof of Theorem 2.11. Let us recall that

$$
\mathcal{D}_{V}^{\delta}:=\left\{x \in \mathbb{R}^{n} \backslash\{0\}: \Psi_{V}(x)<-\delta(x)\right\} .
$$

Since the function $\Psi_{V}$ is upper semicontinuous, the set $\mathcal{D}_{V}^{\delta}$ is open, and since $\delta<V$ on $\mathbb{R}^{n} \backslash\{0\}$, by the same proof as before (see proof of Theorem 2.9) it is dense. Furthermore, by hypothesis (2.9), it is straightforward to show that there exists a continuous positive definite function $\epsilon: \mathbb{R}^{n} \rightarrow \mathbb{R}$ such that

$$
\forall x \in \mathcal{D}_{V}^{\delta}, \quad \delta(x)+\epsilon(x)<V(x)
$$

and

$$
\forall x \in \mathcal{D}_{V}^{\delta}, \quad \Psi_{V}(x) \leq-\delta(x)-\epsilon(x)
$$

This implies, by the same proof as for Theorem 2.9 (replacing the term $\frac{V(x)}{2}$ by $\delta(x)+\epsilon(x))$, that $\mathcal{D}_{V}^{\delta}$ is open dense and that there exists a continuous function

$$
u: \mathcal{D}_{V}^{\delta} \rightarrow U
$$

such that for any $x \in \mathcal{D}_{V}^{\delta}$,

$$
\forall \zeta \in \partial V(x), \quad\langle\zeta, f(x, u(x))\rangle \leq-\delta(x)-\epsilon(x) .
$$

Now, we claim that there exists a function

$$
\bar{u}: \mathcal{D}_{V}^{\delta} \rightarrow U
$$

which is smooth and such that

$$
\forall x \in \mathcal{D}_{V}^{\delta}, \quad\|u(x)-\bar{u}(x)\| \leq \frac{\epsilon(x)}{K_{V}(x) \sum_{i=1}^{m} M_{i}},
$$

where $K_{V}(x)$ denotes the Lipschitz constant of the function $V$ on the ball $\bar{B}\left(x, \frac{\|x\|}{2}\right)$ and where the $M_{i}$ 's are the upper bounds of the functions $f_{i}$ 's on the ball $2\|x\| \bar{B}$. Such a function brings that for any $x \in \mathcal{D}_{V}^{\delta}$ and for any $\zeta \in \partial V(x)$,

$$
\begin{aligned}
\left\langle\zeta, f_{0}(x)+\sum_{i=1}^{m} \overline{u_{i}}(x) f_{i}(x)\right\rangle & \leq-\delta(x)-\epsilon(x)+\|\zeta\|\|u(x)-\bar{u}(x)\| \sum_{i=1}^{m}\left\|f_{i}(x)\right\| \\
& \leq-\delta(x)-\epsilon(x)+K_{V}(x) \sum_{i=1}^{m} M_{i}\|u(x)-\bar{u}(x)\| \\
& \leq-\delta(x) .
\end{aligned}
$$


Finally, considering a Carathéodory solution $x(\cdot)$ of

$$
\dot{x}=f_{0}(x)+\sum_{i=1}^{m} \overline{u_{i}}(x) f_{i}(x)
$$

starting at $x_{0} \in \mathbb{R}^{n}$ we get that for any $t_{0}>0$ such that $x\left(t_{0}\right) \neq 0$,

$$
\Psi_{V}\left(x\left(t_{0}\right)\right) \leq-\delta\left(x\left(t_{0}\right)\right) .
$$

This means by $(2.9)$ that the trajectory $x(\cdot)$ stays in $\mathcal{D}_{V}^{\delta}$ for positive times. Theorem 2.11 is proved.

Acknowledgments. The author is grateful to Francis Clarke for comments and several corrections on a previous version of the paper. He is also indebted to Olivier Ley for his help in the writing of this article.

\section{REFERENCES}

[1] G. Alberti, L. Ambrosio, and P. Cannarsa, On the singularities of convex functions, Manuscripta Math., 76 (1992), pp. 421-435.

[2] A.D. Alexandroff, Almost everywhere existence of the second differential of a convex function and some properties of convex surfaces connected with it, Leningrad State Univ. Annals [Uchenye Zapiski] Math. Ser., 6 (1939), pp. 3-35.

[3] L. Ambrosio, P. Cannarsa, and H.M. Soner, On the propagation of singularities of semiconvex functions, Ann. Scuola Norm. Sup. Pisa Cl. Sci. (4), 20 (1993), pp. 597-616.

[4] F. Ancona And A. Bressan, Patchy vector fields and asymptotic stabilization, ESAIM Control Optim. Calc. Var., 4 (1999), pp. 445-471.

[5] Z. Artstein, Stabilization with relaxed controls, Nonlinear Anal., 7 (1983), pp. 1163-1173.

[6] J.P. Aubin and H. Frankowska, Set-Valued Analysis, Birkhäuser Boston, Boston, 1990.

[7] R.W. Brockett, Asymptotic stability and feedback stabilization, in Differential Geometric Control Theory, R.W. Brockett, R.S. Millman, and H.J. Sussmann, eds., Birkhäuser Boston, Boston, 1983, pp. 181-191.

[8] F.H. Clarke, Optimization and Nonsmooth Analysis, Wiley-Interscience, New York, 1983; reprinted as Classics Appl. Math. 5, SIAM, Philadelphia, 1990.

[9] F.H. Clarke, Yu.S. Ledyaev, L. Rifford, and R.J. Stern, Feedback stabilization and Lyapunov functions, SIAM J. Control Optim., 39 (2000), pp. 25-48.

[10] F.H. Clarke, Yu.S. Ledyaev, E.D. Sontag, and A.I. Subbotin, Asymptotic controllability implies feedback stabilization, IEEE Trans. Automat. Control, 42 (1997), pp. 1394-1407.

[11] F.H. Clarke, Yu.S. Ledyaev, and R.J. Stern, Asymptotic stability and smooth Lyapunov functions, J. Differential Equations, 149 (1998), pp. 69-114.

[12] F.H. Clarke, Yu.S. Ledyaev, R.J. Stern, and P.R. Wolenski, Nonsmooth Analysis and Control Theory, Grad. Texts in Math. 178, Springer-Verlag, New York, 1998.

[13] J.-M. Conon, Global asymptotic stabilization for controllable systems without drift, Math. Control Signals Systems, 5 (1992), pp. 295-312.

[14] M.G. Crandall, H. Ishit, AND P.-L. Lions, User's guide to viscosity solutions of second order partial differential equations, Bull. Amer. Math. Soc. (N.S.), 27 (1992), pp. 1-67.

[15] N.N. Krasovski and A.I. Subbotin, Game-Theoretical Control Problems, Springer-Verlag, New York, 1988.

[16] Yu.S. Ledyaev And L. Rifford, Robust Stabilization of the Nonholonomic Integrator, in preparation.

[17] Yu.S. Ledyaev and E.D. Sontag, A Lyapunov characterization of robust stabilization, Nonlinear Anal., 37 (1999), pp. 813-840.

[18] E. Michael, Continuous selections. I, Ann. of Math. (2), 63 (1956), pp. 361-382.

[19] F. Morgan, Geometric Measure Theory. A Beginner's Guide, Academic Press, Boston, 1988.

[20] J.-B. Pomet, Explicit design of time-varying stabilizing control laws for a class of controllable systems without drift, Systems Control Lett., 18 (1992), pp. 147-158.

[21] L. RIFFORD, On the existence of nonsmooth control-Lyapunov functions in the sense of generalized gradients, ESAIM Control Optim. Calc. Var., 6 (2001), pp. 593-61.

[22] L. Rifford, Singularities of Some Viscosity Supersolutions and the Stabilization Problem in the Plane, manuscript. 
[23] L. RIFFORD, Existence of Lipschitz and semiconcave control-Lyapunov functions, SIAM J. Control Optim., 39 (2000), pp. 1043-1064.

[24] L. Rifford, Problèmes de stabilisation en théorie du controle, Ph.D. thesis, Université Claude Bernard Lyon I, Lyon, France, 2000.

[25] L. Rifford, Stabilisation des systèmes globalement asymptotiquement commandables, C. R. Acad. Sci. Paris Sér. I Math., 330 (2000), pp. 211-216.

[26] E.D. Sontag, A "universal" construction of Artstein's theorem on nonlinear stabilization, Systems Control Lett., 13 (1989), pp. 117-123.

[27] E.D. Sontag, Stability and stabilization: Discontinuities and the effect of disturbances, in Nonlinear Analysis, Differential Equations and Control (Montreal, QC, 1998), Kluwer Academic, Dordrecht, The Netherlands, 1999, pp. 551-598.

[28] E.D. Sontag And H.J. Sussmann, Remarks on continuous feedback, in Proceedings of the IEEE Conference on Decision and Control, Albuquerque, NM, 1980, IEEE, pp. 916-921.

[29] E.D. Sontag And H.J. Sussmann, Nonsmooth control-Lyapunov functions, in Proceedings of the IEEE Conference on Decision and Control, New Orleans, LA, 1995, IEEE, pp. 27992805 .

[30] E.D. Sontag and H.J. Sussmann, General classes of control-Lyapunov functions, in Stability Theory (Ascona, 1995), Birkhäuser, Basel, 1996, pp. 87-96.

[31] H.J. Sussmann, Subanalytic sets and feedback control, J. Differential Equations, 31 (1979), pp. $31-52$.

[32] L. ZAJÍČEK, On the differentiation of convex functions in finite and infinite dimensional spaces, Czechoslovak Math. J., 29 (1979), pp. 340-348. 